Doroshenko S. I., Savonik S. M. Peculiarities of the prevalence of partial secondary adentia and its complications in children and adolescents. Journal of Education, Health and Sport. 2020;10(12):178-184. eISSN 2391-8306. DOI http://dx.doi.org/10.12775/JEHS.2020.10.12.017

https://apcz.umk.pl/czasopisma/index.php/JEHS/article/view/JEHS.2020.10.12.017

https://zenodo.org/record/4386034

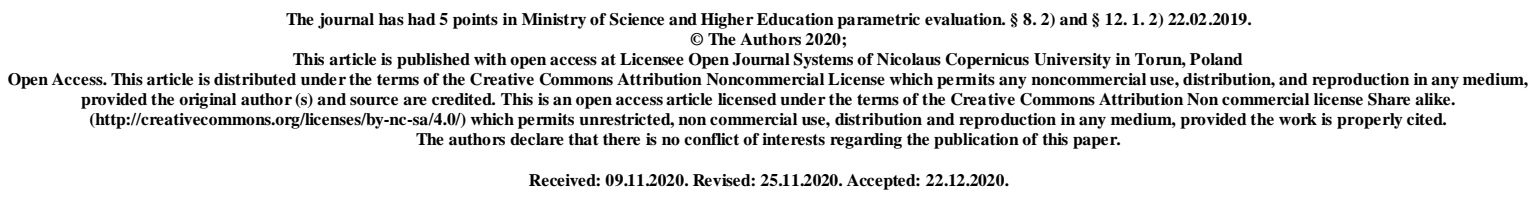

UDK 616.314-007.24-053.2-084-08

\title{
PECULIARITIES OF THE PREVALENCE OF PARTIAL SECONDARY ADENTIA AND ITS COMPLICATIONS IN CHILDREN AND ADOLESCENTS
}

\author{
S. I. Doroshenko, S. M. Savonik
}

\section{Private Educational Institution "Kyiv Medical University"}

Doroshenko S.I. - doctor of medical sciences, professor, head of the department of orthopedic dentistry and orthodontics, Private Educational Institution "Kyiv Medical University" 02099, Boryspilska str., 2, Kyiv, Ukraine, Tel: 38 (063) 705-47-48, E-mail: dr.lanasavonik@ gmail.com Savonik S.M. - Assistant of the Department of Orthopedic Dentistry and Orthodontics of the private higher educational institution "Kyiv Medical University" 02099, Boryspilska str., 2, Kyiv, Ukraine, Tel: 38 (063) 705-47-48, E-mail: dr.lanasavonik@ gmail.com

\section{Abstract}

Rationale. The high prevalence of anomalies, partial adentia and its complications determine the importance of searching new optimal means of prevention, orthodontic and orthopaedic treatment, taking into account the age peculiarities of a child's body during its formation.

Objective: To study the prevalence, etiological factors and features of the clinical progression of partial secondary adentia and its complications in children and adolescents in order to develop optimal means of prevention, orthodontic and orthopaedic treatment.

Materials and methods. 2276 people aged from 4 to 17 years were examined during the temporary, mixed and permanent dentition periods, in order to study the prevalence of partial secondary adentia and its complications in children and adolescents. The examinations 
were conducted in the organized child care of Kyiv (kindergartens, schools) and at the Department of Orthopedic Dentistry and Orthodontics of the private higher educational institution "Kyiv Medical University". All patients were divided into 3 age groups depending on the period of formation of the dento-maxillaire system.

Results. According to the results of the examinations, the high prevalence of partial secondary adentia was detected in both posterior and anterior dentition, in particular, among the examined 2276 children, partial secondary adentia was detected in 359 people, including 115 children with the defect of the anterior area and 264 people had the defect of posterior dentition.

The causes of partial secondary adentia in the examined children were: the removal of temporary and permanent teeth caused by caries and its complications - 278 (77.4\%) cases, the second place takes - the loss of teeth caused by traumatic injuries - 34 children $(9.3 \%)$.

Deformity of dental arches was diagnosed in 278 people $(77,43 \%)$ among the children with partial secondary adentia, indicating the high prevalence of such a pathology due to untimely dental care, in particular, the lack of prosthetic treatment.

Conclusions. The occurrence of partial secondary adentia in children and adolescents caused by caries and its complications, as well as traumatic injuries in $77.4 \%$ of cases in the absence of prosthetic treatment leads to complications in the form of deformities of dental arches. Carrying out temporary teeth maintenance procedure until the term of their physiological change, as well as taking timely treatment, prevention and prosthetic measures will aid the harmonious development of a child's dento-maxillaire system and prevention of complications.

\section{Keywords. Teeth; partial secondary adentia; deformities of dental arches; caries.}

Rationale. Among the factors that ensure the harmonious growth and development of a child, the physiological development of the dento-maxillaire system is important. Its functioning depends on carrying out the maintenance procedure of teeth of the temporary and permanent occlusion. However, monitoring dental morbidity rate in children of Ukraine indicates the high prevalence of dento-maxillaire deformities, defects of teeth and dentitions, which does not tend to decrease [1,2].

Analyzing the etiological factors of partial adentia, the researchers concluded that caries and its complications take the first place in children, which is $57.6 \%$. Caries most often destroys frontal teeth (53\%), which are of great importance in terms of formation of sounds and facial esthetics. The first molars are less damaged (29\%), which leads to the reduction of 
occlusal vertical dimension and the formation of a short dental arch with its early loss. Other causes include acute and chronic trauma of the dento-maxillaire system, iatrogenic factors, the absence of one or more immature teeth, or their loss at an early age [3 - 6]

The frequency of dentition defects caused by traumatic injury ranges from 2.1 to $8 \%$. Traumatic tooth injury is more common in boys (17.3\% of cases) than in girls $(8 \%)$. The central incisors of the upper jaw, then the lower jaw are more often injured, then the lateral incisors of both jaws, less often - the canines. Thus, of the $12.5 \%$ of traumatic dental injuries in children, $8.1 \%$ are central incisors, $4.1 \%$ are lateral incisors, and only $0.3 \%$ are canines. The rate of acute trauma prevails over chronic one and accounts for about $32 \%$ of cases at an early age. [1,9].

The dentition defect could result from congenital absence of immature teeth congenital adentia. Congenital adentia ranks the third place and reaches $1,2-4 \%$ by frequency of occurrence $[8,9]$.

According to the researchers, the deformities of the dentition at an early age are significantly different from the corresponding deformities that develop among adults. The peculiarities lie in the fact that at an early age, teeth that do not have antagonists are displaced towards the defect together with the alveolar process, there is also a migration of permanent immature teeth in the bone. Sometimes, one year is enough for severe morpho-functional changes of a child's dento-maxillaire system. $[1,7,9,10]$.

Timely prevention of premature removal of temporary teeth in children through preventive measures aimed at preventing caries and its complications is of great importance for achieving positive dynamics in pediatric dentistry. If conservative treatment does not work and you have to remove temporary teeth, you should proceed with their replacement.

The study of the prevalence, etiological factors and features of the clinical progression of dentition defects in children and adolescents will contribute to the development of methods for optimizing the harmonious development of their dento-maxillaire system, which determines the rationale of our research.

Objective: To study the prevalence, etiological factors and features of the clinical progression of partial secondary adentia and its complications in children and adolescents in order to develop optimal means of prevention, orthodontic and orthopaedic treatment.

\section{Material and methods}

We examined 2276 people aged from 4 to 17 years during the temporary, mixed and permanent dentition period in order to study the prevalence of partial secondary adentia and its complications in children and adolescents during 2016 - 2020. The examinations were 
conducted in organized child care of Kyiv (kindergartens, schools) and at the Department of Orthopedic Dentistry and Orthodontics of the private higher educational institution "Kyiv Medical University". There were 1083 boys and 1193 girls among the examined children. All patients were divided into 3 age groups depending on the period of formation of the dentomaxillary system: the first group included children $4-5$ years, the second - $6-13$ years and the third - $14-17$ years, respectively.

The examination was conducted according to the common method using disposable instruments. The obtained data was added to a specially designed chart.

During the examination, the hygienic condition of the state of the oral cavity was diagnosed using the index of Yu. A. Fedorov and V.V. Volodkina, gingivitis was diagnosed using the PMA index and the presence of caries cavities in the teeth, the degree of their damage. Partial secondary adentia and its complications, topography of dentition defects, number of missing teeth, presence of anomalies and deformities, as well as anomalies of shape and position of individual teeth, occlusion condition were detected. The causes of early teeth loss and the presence of bad health habits were diagnosed. Additional methods of examination were used in the treatment. They were anthropometric measurements of diagnostic models of the jaws by the method of Pont, Korkhaus. X-ray - lateral orthopantomograms and teleradiograms. Partial adentia was systematized by length and localization. In the absence of 1 tooth - the dentition defect was considered small, in the absence of 2 - 3 teeth - medium, and the absence of more than 3 teeth - major. According to localization, they were divided into: dentition defect in the anterior area and in the posterior area.

\section{Results of original research}

According to the results of the clinical examination of children and adolescents, aged 4 - 17 years, three age groups were formed: the first group included children $4-5$ years, the second - 6-13 years and the third - adolescents $14-17$ years, respectively, as well, they were divided by gender.

Among the examined 2,276 children, females predominated (52.42\%), and males accounted for $47.58 \%$. The largest number of the examined children - aged from 6 to 13 years $(78.86 \%)$.

Special attention was paid to the topography of the dentition defects during our examination with partial adentia: in the anterior and posterior areas, complicated or uncomplicated by deformities of the dental arches. 
Partial secondary adentia was diagnosed in 359 people, including 203 boys and 156 girls, among 2,276 examined children. A characteristic feature was the gradual increase in the prevalence of partial secondary adentia in the age-related aspects. In particular, partial secondary adentia was diagnosed in 20 schoolchildren $(5.57 \%)$ in the mixed dentition period among 6 years old children, children aged 7 years - 69 (19.22\%) children, children aged 8 years - $86(23,95 \%)$, and at the age of 9 and 10 years - $45(12.53 \%)$ and $49(13.65 \%)$ cases, respectively. A decrease in the number of children with partial secondary adentia was observed at the end of the mixed dentition period, and at the beginning of the permanent one. In particular, this pathology was diagnosed in 17 people among 11-year-old children, which is $4.7 \%$, and in 12-year-olds among 12 schoolchildren (3.3\%), respectively.

Among the examined children with permanent occlusion, partial secondary adentia was diagnosed: at the age of 13 - in 14 people (3.9\%), at 15 years - in 12 people (3.3\%), at 16 years - in 7 people $(1,9 \%)$ and at the age of 17 in 9 children $(2.5 \%)$.

The main cause of partial secondary adentia in the examined children was the removal of temporary and permanent teeth due to caries and its complications - $278(77.4 \%)$ cases, as well as tooth loss caused by traumatic injuries - 34 children (9.3\%). Partial adentia of unknown etiology was diagnosed in 47 students (due to the inability to collect a complete medical history).

The examined 359 children with secondary partial adentia were diagnosed with the presence of small, medium and major defects of the dentition. In particular, small defects were observed in 223 people (62.1\%): in the temporary dentition period - 4 (1.8\%); in the mixed - 168 (75.3\%); in the permanent - 52 (22.9\%). Medium defects occurred in 96 children (26.7\%): in the temporary dentition period - $3(3.1 \%)$; in the mixed - $82(85.4 \%)$; and in the permanent - $13(13.5 \%)$, respectively. Major defects occurred in 26 children (7.24\%): in the temporary dentition period $-1(3.8 \%)$; in the mixed - $22(84.6 \%)$; in the permanent -13 (11.6\%), respectively.

Partial secondary adentia of the anterior part of the dentition was detected in 115 children, which accounted for $32.03 \%$ of all cases of this pathology. The prevalence of this pathology had its characteristics associated with the periods of occlusion, in particular in the temporary dentition period partial secondary adentia of the anterior dentition was observed in only 3 people $(2.6 \%)$ of all cases, in the mixed - in 93 people (80.9\%), and in the permanent in 19 people, which amounted to $16.5 \%$, respectively.

A higher prevalence of partial secondary adentia was in the posterior areas of the dentition, which was observed in 264 children, accounting for $72.7 \%$ of the total number 
examined; in the anterior area - 115 people (32.0\%), respectively. In the mixed dentition period, partial secondary adentia occurred in 208 cases $(79.7 \%)$, and the permanent - in 93 people $(80.9 \%)$, respectively.

Deformities of the dental arches were diagnosed in $77.43 \%$ (278) among the examined children who have partial secondary adentia, which indicates the high prevalence of this pathology due to untimely dental care, in particuar the lack of prosthetic treatment.

Thus, the results of the examination of a representative cohort of people indicates the high prevalence of dental pathology among children of different ages. Timely prevention of premature removal of temporary teeth in children through the preventive measures aimed at inhibiting caries and its complications will help to achieve positive dynamics in pediatric dentistry, and further study of the prevalence, etiology and clinical progression of partial adentia in children and adolescents will aid the design of methods for optimizing the harmonious development of their dento-maxillaire system.

\section{Conclusions}

1. The results of the clinical examination of a representative cohort of children and adolescents indicate the significant prevalence of partial secondary adentia, both in the posterior and asterior areas. Partial secondary adentia was detected in 359 people among 2276 children, which reached up to $15.9 \%$, of which 115 children had this pathology of the anterior part of the dentition and 264 people - in the posterior part.

2. Among the etiological factors of partial secondary adentia in the examined children the most significant were: removal of temporary and permanent teeth caused by caries and its complications - 278 (77.4\%), and loss of teeth caused by traumatic injuries - 34 children, $(9.3 \%)$ cases.

3. Among children with partial secondary adentia, deformities of the dental arches were diagnosed in 278 people (77.43\%), which indicates the high prevalence of this pathology due to untimely dental care, in particular the lack of prosthetic treatment.

4. In the harmonious growth and development of a child's dento-maxillaire system, the most important role belongs to carrying out the maintenance procedure of temporary teeth until their physiological change by permanent teeth.

\section{Perspectives of further developments}

1. The study of the prevalence, etiological factors and features of the clinical progression of partial secondary adentia in children and adolescents will contribute to the development of methods for maintaining dental health and harmonious development of the dento-maxillaire system. 


\section{References}

1. Doroshenko S.I. Optimization of orthopedic treatment of patients with defects of teeth and dentitions complicated by secondary dental deformities / SI Doroshenko, OV Fedorova, SV Irkha, E. Elmaghrabi, AV Storozhenko // Visnyk stomatologii. - 2019. - V. 32, № 2. - P. 38-42.

2. Zayats O.R., Ozhogan Z.R. Prevalence of dento-maxillaire anomalies in children Ivano-Frankivsk region. Modern dentistry 1/2020 p.68-72

3. Bida O.V., Ozhogan Z.R. Features of orthodontic treatment of dental deformities due to partial loss of teeth, depending on the bone density of the jaws. Innovations in dentistry. Scientific and practical electronic journal 3-4 (16): 30-35. 2017

4. Mirchuk B.M., Maksimov Y.V. Frequency of the dentition defects among adult patients of Zaporozhye who applied for prosthetic treatment. Current issues of pharmaceutical and medical science and practice volume 101 (23): 102 -106 2017

5. Smaglyuk L.V. Dental status of young people of different somatotypes / LV Smaglyuk, DV Sheshukov // Bulletin of problems of biology and medicine. - 2018. - Vip. 1 (2). - P. 365-369

6. Storozhenko K.V. Peculiarities of treatment of prenormal forms of occlusion in different periods of formation of the dento-maxillaire system (literature review) / Storozhenko KV // Dental Science and Practice. - 2016. - No2 (13) - P. 38-43.

7. Drogomiretskaya M.S., Yakimets A.V., Leporsky D.V. Results of biometric examination of patients with congenital adentia of lateral incisors of the upper jaw. Collection of scientific works of NMAPE named after PL Shupika, 462-467 2016

8. Makhnytsky D.M. Secondary dental deformities in children, their prevention and treatment. Scientific Journal «ScienceRise» №5 / 4 (10) 2015

9. Kovach I.V., Lavreniuk Y.V. General characteristics of dental morbidity rate in children on the background of orthodontic treatment. Honey. perspectives. 2016; 21 (1): 1048

10. Nespryadko V.P., Kyryliuk V.V. Influence of partial loss of teeth and fixed dentures on organs and tissues of the oral cavity. Bulletin of problems of biology and medicine 1 (117): 13-18. Available http://vpbm.com.ua/upload/2015-1/4.pdf. 12/12/2015 